\title{
Entanglement and the Speed of Evolution of Two Interacting Qubits
}

\author{
C. Zander ${ }^{1}$, A. Borras ${ }^{2}$, A.R. Plastino ${ }^{2,4,5}$, A. Plastino ${ }^{5}$, and M. Casas ${ }^{2,3}$ \\ ${ }^{1}$ Physics Department, University of Pretoria, Pretoria 0002, South Africa \\ ${ }^{2}$ Departament de Fisica, Universitat de les Illes Balears, 07122 Palma de Mallorca, Spain \\ ${ }^{3}$ Institute for Cross-Disciplinary Physics and Complex Systems, \\ IFISC (CSIC-UIB, Palma de Mallorca 07122, Spain) \\ ${ }^{4}$ Instituto Carlos I de Física Teórica y Computacional, \\ Universidad de Granada, Granada, Spain \\ ${ }^{5}$ CREG-National University La Plata-Conicet, \\ C.C. 727, 1900 La Plata, Argentina
}

(Dated: January 11, 2013)

\begin{abstract}
The time needed by a quantum system to reach a state fully distinguishable from the original one provides a natural way of determining how fast the corresponding dynamical evolution is. This orthogonality time admits a lower bound, expressible in terms of the energy's expectation value and the energy's standard deviation, that yields a "quantum speed limit". Composite quantum systems need entanglement in order to achieve this limit. So far, most studies on the connection between entanglement and the quantum speed limit have focused on the case of non-interacting systems. The connection between quantum speed and entanglement is systematically investigated here for the case of a system of two interacting qubits, taking into consideration all possible initial states that evolve into an orthogonal one.
\end{abstract}

keywords: Quantum Entanglement, Quantum Evolution, Time-Energy Uncertainty Relations PACS numbers: 03.65.-w; 03.65.Ud; 03.67.-a 


\section{INTRODUCTION}

Two pure states of a quantum system are fully distinguishable if, and only if, they are orthogonal. This basic fact of quantum physics leads to a natural way of assessing how fast a quantum system evolves in time [1-4]. The "speed" of quantum evolution is determined by the minimum time $\tau$ required by a quantum system to evolve into a state orthogonal to the initial one (the smaller the orthogonality time $\tau$ is, the faster is the system's evolution). This approach to the speed of quantum dynamical evolution is relevant from the fundamental as well as the applied points of view. The orthogonality time of a quantum system with Hamiltonian $H$ admits lower bounds both in terms of the standard deviation $\Delta E=\sqrt{\left\langle H^{2}\right\rangle-\langle H\rangle^{2}}$ of the energy and also in terms of the shifted energy expectation value $E=\langle H\rangle-E_{0}$, where $E_{0}$ is the energy of the system's ground state; that is, when computing $E$ one chooses the zero of energy as the energy of the ground state. The inequality relating $\tau$ to $\Delta E$,

$$
\Delta E \tau \geq \frac{1}{2} \pi \hbar
$$

can be interpreted as a time-energy uncertainty relation [5]. This is not, of course, the only possible formulation of a time-energy uncertainty relation. The study of such relations and their applications is in itself an active research area (see, for instance, [5, 6] and references therein). An alternative lower bound for the orthogonality time, based directly on the energy expectation value, was discovered by Margolus and Levitin [1],

$$
E \tau \geq \frac{1}{2} \pi \hbar
$$

When considering quantum mechanical information processing devices, the orthogonality time $\tau$ provides a convenient estimate of the minimum time required by the system to perform an elementary information processing step. A considerable amount of research has been devoted in recent years to the investigation of this and related concepts, which constitute powerful tools for exploring the fundamental limits imposed by the quantum mechanical laws of Nature on the information-processing capabilities of physical systems $[7-16]$.

Combining the two basic lower bounds for the orthogonality time, one based on $\Delta E$ and the other one on $E$, the lowest possible value of $\tau$ is [3] 


$$
\tau_{\min }=\max \left(\frac{\pi \hbar}{2 E}, \frac{\pi \hbar}{2 \Delta E}\right) .
$$

Giovannetti et al. discovered an interesting relation between the quantum speed limit given by (3) and entanglement [3]. It turns out that in the case of composite quantum systems entanglement is essential for achieving the minimum orthogonality time $\tau_{\min }$. Nonentangled (pure) initial states whose evolution is described by a non-interacting Hamiltonian (which can't generate entanglement) do not achieve the speed limit; except in marginal cases where only one of the subsystems actually evolves, and the rest are in eigenstates of their respective Hamiltonians.

In order to reach the speed limit in situations where all the subsystems do really evolve it is necessary that either: (i) the initial state of the system is entangled or (ii) the Hamiltonian is endowed with an interaction term that generates entanglement as the quantum evolution takes place. The fulfilment of at least one of these requirements is a necessary (but not sufficient) condition to achieve the maximum speed allowed by quantum mechanics.

The connection between entanglement and the quantum speed limit has been the subject of various recent research works [7, 17-21]. Many of these efforts focused on the case of non-interacting systems. In particular, in [20] a systematic exploration of the quantum speed-entanglement connection for systems of two non-interacting qubits was conducted, taking into account all possible initial pure states exhibiting a finite orthogonality time. In this study [20] the case of systems of two identical particles (either fermions or bosons) was also considered. In [21] the relation between quantum speed and entanglement was investigated for general mixed states of systems of two non-interacting qubits. Some aspects of the role of entanglement in the speed of evolution of systems of $N$ non-interacting qubits were considered in [19]. The entanglement-related aspects of the quantum speed of a system of two identical fermions evolving according to a Hubbard-like Hamiltonian was investigated in [17]. A reformulation of the connection between quantum speed and entanglement based upon the quantum Fisher information, focusing on the case of non-interacting subsystems, was advanced in [7]. The concept of quantum brachistochrone [22-26] has been used to elucidate some of the entanglement features exhibited by general time-optimal quantum evolutions (comprising both interacting and non-interacting scenarios) [27-30]. However, the detailed characterization of the connection between quantum speed and entanglement for interacting composite systems is a problem that still remains largely unexplored. 
Appropriate multi-qubit interacting Hamiltonians can generate entanglement in such a way that an initially separable state can reach the minimum orthogonality time $\tau_{\min }$ [3]. To achieve this task two different conditions must be fulfilled: (i) all the qubits must be connected by the interaction and (ii) the interaction must be sufficiently strong. The quantum speed of evolution associated with this type of Hamiltonian has been studied only in the case of particular families of initial states. The typical behavior of more general classes of states still remains unknown. The aim of the present work is to explore the connection between entanglement and quantum speed for general two-qubit states that reach an orthogonal state when evolving according to a paradigmatic entangling Hamiltonian of relevance for information-related quantum technologies.

\section{QUANTUM SPEED AND ENTANGLEMENT IN A SYSTEM OF TWO IN- TERACTING QUBITS}

We are considering a system of two interacting qubits evolving according to the Hamiltonian

$$
H=\hbar \omega_{0}\left[2 I^{(1)} \otimes I^{(2)}-\sigma_{x}^{(1)} \otimes I^{(2)}-I^{(1)} \otimes \sigma_{x}^{(2)}\right]+\hbar \omega\left[I^{(1)} \otimes I^{(2)}-\sigma_{x}^{(1)} \otimes \sigma_{x}^{(2)}\right],
$$

where $I$ is the identity matrix and $\sigma_{x}$ is the $x$-Pauli operator. The Hamiltonian (4) depends on two parameters with the dimension of frequency, $\omega_{0}$ and $\omega$. The first parameter characterizes the free Hamiltonian of the qubits (first term in (4)) and the parameter $\omega$ is associated with the interaction (second term in (4)). The eigenvectors of $H$ are

$$
\begin{aligned}
\left|u_{0}\right\rangle & =\frac{1}{2}\{|00\rangle+|01\rangle+|10\rangle+|11\rangle\}, \\
\left|u_{1}\right\rangle & =\frac{1}{2}\{|00\rangle-|01\rangle-|10\rangle+|11\rangle\}, \\
\left|u_{2}\right\rangle & =\frac{1}{\sqrt{2}}\{-|00\rangle+|11\rangle\}, \\
\left|u_{3}\right\rangle & =\frac{1}{\sqrt{2}}\{-|01\rangle+|10\rangle\},
\end{aligned}
$$

with the concomitant eigenvalues respectively being $\left\{0,4 \hbar \omega_{0}, 2 \hbar\left(\omega+\omega_{0}\right), 2 \hbar\left(\omega+\omega_{0}\right)\right\}$. The Hamiltonian (4) describes systems that have technological relevance because the interaction 
part is closely related to a Hamiltonian leading to a time evolution that can substantially increase the power-limited communication capacity of coupled quantum channels (see [31]). It is clear that the time evolution associated with the Hamiltonian (4) is not described by a local unitary operation. It is, instead, given by a global unitary transformation that cannot be factorized into local parts. This implies that the amount of entanglement exhibited by the bipartite system is time dependent.

As already mentioned, it is possible for an appropriate interaction to build up entanglement so that the system can reach the speed bound even though no entanglement is present initially. In fact, it was shown in [3] that an interaction like the one in (4) is indeed capable of speeding up the dynamics of initially non-entangled states. However, that study was limited only to a particular set of initial states. The purpose here is to get a more complete picture of the connection between entanglement and quantum speed for a Hamiltonian of the aforementioned form. It must be emphasized that our main focus in the present work is not the absolute time $\tau$ required to reach an orthogonal state, but the ratio $\tau / \tau_{\text {min }}$ between $\tau$ and the lower bound $\tau_{\text {min }}$ determined by the system's energy $E$ and energy variance $\Delta E$ (see equation (3)). It is instructive, however, to consider the values of $\tau_{\text {min }}$ corresponding to those initial states that reach an orthogonal state in the shortest possible time when evolving according to the Hamiltonian (4). These states have the smallest possible value of the orthogonality time and they saturate the $(E, \Delta E)$-based bound (having thus $\tau=\tau_{\min }$ ). These states have the form,

$$
\begin{array}{ll}
a_{0}\left|u_{0}\right\rangle+a_{2}\left|u_{2}\right\rangle+a_{3}\left|u_{3}\right\rangle, & \text { with }\left|a_{0}\right|^{2}=\left|a_{2}\right|^{2}+\left|a_{3}\right|^{2}=\frac{1}{2}, \quad\left(\omega>\omega_{0}\right), \\
a_{0}\left|u_{0}\right\rangle+a_{1}\left|u_{1}\right\rangle+a_{2}\left|u_{2}\right\rangle+a_{3}\left|u_{3}\right\rangle, & \text { with }\left|a_{0}\right|^{2}=\left|a_{1}\right|^{2}+\left|a_{2}\right|^{2}+\left|a_{3}\right|^{2}=\frac{1}{2}, \quad\left(\omega=\omega_{0}\right), \\
a_{0}\left|u_{0}\right\rangle+a_{1}\left|u_{1}\right\rangle, & \text { with }\left|a_{0}\right|^{2}=\left|a_{1}\right|^{2}=\frac{1}{2}, \quad\left(\omega<\omega_{0}\right),
\end{array}
$$

and the corresponding values of the orthogonality times are,

$$
\begin{array}{ll}
\tau=\tau_{\min }=\frac{\pi}{2\left(\omega+\omega_{0}\right)}, & \left(\omega \geq \omega_{0}\right), \\
\tau=\tau_{\min }=\frac{\pi}{4 \omega_{0}}, & \left(\omega<\omega_{0}\right) .
\end{array}
$$

When studying the connection between quantum speed and entanglement, Giovannetti et al. considered a separable initial state, and then analyzed the effect of varying $\omega$ and $\omega_{0}$. 
We shall consider, instead, general families of initial states endowed with finite orthogonality times, and restrict ourselves to specific cases of $\omega$ and $\omega_{0}$ admitting an analytical treatment. For these families of initial states of the two-qubit system, we are going to investigate the connection between the quotient $\tau / \tau_{\min }$ (which determines the speed of quantum evolution) and the amount of entanglement $\mathcal{E}$ exhibited by those states. As a measure of entanglement of the two-qubit system we shall use the linear entropy of the marginal density matrix $\rho_{1}$ corresponding to one of the qubits,

$$
\mathcal{E}=2\left(1-\operatorname{Tr}\left(\rho_{1}^{2}\right)\right)
$$

where $\rho_{1}=\operatorname{Tr}_{2} \rho$, and $\rho=\left|\psi\left(t_{0}\right)\right\rangle\left\langle\psi\left(t_{0}\right)\right|$ is the joint density matrix globally describing the composite system. The linear entropy of a quantum density matrix is a useful measure with multiple applications. It has the important advantage that in order to to evaluate it one does not need to compute first the eigenvalues of the density matrix. It is closely related to measures of purity of quantum states, based on the trace of the squared density matrix [32]. The inverse of the trace of the squared marginal density matrix associated with a subsystem gives the (effective) Schmidt number of a pure state describing a bipartite quantum system [33-35]. The linear entropy of the marginal density matrix of a subsystem has been recently used by several researchers as a convenient entanglement measure for pure states of bipartite quantum systems (see, for instance [36]).

In what follows we shall explore the connection between entanglement and the speed of quantum evolution considering both the amount of entanglement exhibited by initial states evolving into an orthogonal one, and the time averaged entanglement associated with such an evolution. We consider these two quantities because they constitute estimates of the amount of entanglement, regarded as a resource, needed by the system in order to evolve into a state fully distinguishable from the initial one. We are interested in the "entanglement cost" of evolving into an orthogonal state, and in how this "cost" is related to the quantum speed. When focusing upon the initial entanglement one deals with the amount of entanglement that has to be generated when preparing the initial state. On the other hand, the time averaged entanglement provides us with a global estimate of the amount of entanglement involved in the full evolution, including the entanglement buildup by the interaction.

Let us consider a general initial state at time $t_{0}$ given by 


$$
\begin{aligned}
\left|\psi\left(t_{0}\right)\right\rangle= & a_{0}\left|u_{0}\right\rangle+a_{1}\left|u_{1}\right\rangle+a_{2}\left|u_{2}\right\rangle+a_{3}\left|u_{3}\right\rangle \\
= & {\left[\frac{1}{2} a_{0}+\frac{1}{2} a_{1}-\frac{1}{\sqrt{2}} a_{2}\right]|00\rangle+\left[\frac{1}{2} a_{0}-\frac{1}{2} a_{1}-\frac{1}{\sqrt{2}} a_{3}\right]|01\rangle } \\
& +\left[\frac{1}{2} a_{0}-\frac{1}{2} a_{1}+\frac{1}{\sqrt{2}} a_{3}\right]|10\rangle+\left[\frac{1}{2} a_{0}+\frac{1}{2} a_{1}+\frac{1}{\sqrt{2}} a_{2}\right]|11\rangle,
\end{aligned}
$$

with

$$
\left|a_{0}\right|^{2}+\left|a_{1}\right|^{2}+\left|a_{2}\right|^{2}+\left|a_{3}\right|^{2}=1
$$

At time $t$ the state will have evolved to

$$
\left|\psi\left(t_{0}+t\right)\right\rangle=a_{0}\left|u_{0}\right\rangle+a_{1} e^{-4 i \omega_{0} t}\left|u_{1}\right\rangle+a_{2} e^{-2 i\left(\omega_{0}+\omega\right) t}\left|u_{2}\right\rangle+a_{3} e^{-2 i\left(\omega_{0}+\omega\right) t}\left|u_{3}\right\rangle .
$$

To obtain all possible states evolving to an orthogonal state we have to find all the possible sets of coefficients $a_{i}$ satisfying

$$
\left\langle\psi\left(t_{0}\right) \mid \psi\left(t_{0}+\tau\right)\right\rangle=\left|a_{0}\right|^{2}+e^{-4 i \omega_{0} \tau}\left|a_{1}\right|^{2}+e^{-2 i\left(\omega_{0}+\omega\right) \tau}\left[\left|a_{2}\right|^{2}+\left|a_{3}\right|^{2}\right]=0,
$$

where $\tau$ is the time needed to reach an orthogonal state. This is a transcendental equation which cannot be solved analytically in the general case (that is, for arbitrary values of $\omega_{0}$ and $\omega$ ), so we shall concentrate on the three cases (A) $\omega=\omega_{0} ;(B) \omega_{0}=0 ;$ (C) $\omega=3 \omega_{0}$. In the first two cases equation (15) is a linear equation in either $e^{-4 i \omega_{0} \tau}$ or $e^{-2 i \omega \tau}$. In the third case we have a quadratic equation in $e^{-4 i \omega_{0} \tau}$. Note that other cases given by $\omega=n \omega_{0}$, with $n$ an integer, lead in general to polynomial equations in $e^{-4 i \omega_{0} \tau}$ that cannot be solved by radicals, except for the cases $n=5$ and $n=7$, respectively yielding cubic and quartic equations. In these cases, the analytical expressions involved are too cumbersome to lead to a really illuminating discussion. Situations with more general values of the quotient $\omega / \omega_{0}$ are better dealt with by recourse to a semi-numerical treatment, as discussed in Section III. Next we are going to separately consider each one of the aforementioned analytically soluble situations.
A. $\omega=\omega_{0}$

In this case the eigenenergies become $\left\{0,4 \hbar \omega_{0}, 4 \hbar \omega_{0}, 4 \hbar \omega_{0}\right\}$ and from eq. (15) we obtain 


$$
\begin{aligned}
\left|a_{0}\right|^{2} & =\left|a_{1}\right|^{2}+\left|a_{2}\right|^{2}+\left|a_{3}\right|^{2}, \\
\tau & =\frac{\pi}{4 \omega_{0}},
\end{aligned}
$$

which, together with the normalization condition, gives

$$
\begin{aligned}
& a_{0}=\left|a_{0}\right|=\frac{1}{\sqrt{2}}, \\
& a_{1}=e^{i \gamma_{1}} \sqrt{\frac{\delta_{1}}{2}}, \\
& a_{2}=e^{i \gamma_{2}} \sqrt{\frac{\delta_{2}\left(1-\delta_{1}\right)}{2}}, \\
& a_{3}=e^{i \gamma_{3}} \sqrt{\frac{\left(1-\delta_{1}\right)\left(1-\delta_{2}\right)}{2}} .
\end{aligned}
$$

We can omit the phase for $a_{0}$ because we can choose an arbitrary global phase such that this coefficient is real, and we have $0 \leq \delta_{1}, \delta_{2} \leq 1$ and $0 \leq \gamma_{1}, \gamma_{2}, \gamma_{3}<2 \pi$. The mean energy $E$ and the dispersion $\Delta E$ are then both equal to $2 \hbar \omega_{0}$, thus giving rise to

$$
\tau_{\min }=\frac{\pi}{4 \omega_{0}}=\tau
$$

Hence all the states evolving to an orthogonal state evolve at the speed limit. To determine the range of entanglement values adopted by these states let us consider, for example, the subset of those states having $\gamma_{1}, \gamma_{2}, \gamma_{3}=0$ (to determine the alluded range of entanglement values it is enough to consider these states because they already "saturate" the full range of possible values between zero and one). The expression for the entanglement of these initial states becomes

$$
\mathcal{E}\left(\delta_{1}, \delta_{2}\right)=\frac{1}{4}\left(1+2 \sqrt{\delta_{1}}-2 \delta_{2}+\delta_{1}\left(-1+2 \delta_{2}\right)\right)^{2} .
$$

According to this expression the entanglement $\mathcal{E}$ can adopt any value between zero and one. In other words, within the set of states that achieve the quantum speed limit, all possible degrees of initial entanglement are represented. Non-entangled states are obtained when setting $\delta_{1}=0$ and $\delta_{2}=\frac{1}{2}$, while maximally entangled states are obtained for $\delta_{1}=1$. 
B. $\omega_{0}=0$

This case is similar to the former case, with the eigenenergies now being $\{0,0,2 \hbar \omega, 2 \hbar \omega\}$ and

$$
\begin{aligned}
\left|a_{0}\right|^{2}+\left|a_{1}\right|^{2} & =\left|a_{2}\right|^{2}+\left|a_{3}\right|^{2}, \\
\tau & =\frac{\pi}{2 \omega} .
\end{aligned}
$$

This gives

$$
\begin{aligned}
& a_{0}=\left|a_{0}\right|=\sqrt{\frac{\delta_{1}}{2}}, \\
& a_{1}=e^{i \gamma_{1}} \sqrt{\frac{1-\delta_{1}}{2}}, \\
& a_{2}=e^{i \gamma_{2}} \sqrt{\frac{\delta_{2}}{2}}, \\
& a_{3}=e^{i \gamma_{3}} \sqrt{\frac{1-\delta_{2}}{2}} .
\end{aligned}
$$

Once again

$$
\frac{\tau}{\tau_{\min }}=1
$$

since $E=\Delta E=\hbar \omega$ gives $\tau_{\min }=\frac{\pi}{2 \omega}$. As in the previous case, to determine the range of possible entanglement values adopted by these states it is sufficient to consider initial states with $\gamma_{1}, \gamma_{2}, \gamma_{3}=0$. This leads to

$$
\mathcal{E}\left(\delta_{1}, \delta_{2}\right)=\delta_{1}\left(1-\delta_{1}\right)+\frac{1-2 \delta_{2}}{4}\left(\left(1-2 \delta_{2}\right)+4 \sqrt{\delta_{1}\left(1-\delta_{1}\right)}\right) .
$$

Thus, in this case we also have initial states with all possible values of entanglement between zero and one. Separable states are obtained for $\delta_{1}=0,1$ and $\delta_{2}=\frac{1}{2}$ and maximally entangled states for $\delta_{1}=\frac{1}{2}$ and $\delta_{2}=0$.

C. $\omega=3 \omega_{0}$

The eigenenergies are now $\left\{0,4 \hbar \omega_{0}, 8 \hbar \omega_{0}, 8 \hbar \omega_{0}\right\}$ and thus eq. (15), whose roots determine the orthogonality time, becomes 


$$
\begin{aligned}
P(x) & =\left|a_{0}\right|^{2}+\left|a_{1}\right|^{2} x+\left[\left|a_{2}\right|^{2}+\left|a_{3}\right|^{2}\right] x^{2}=0, \\
x & =e^{-4 i \omega_{0} \tau} .
\end{aligned}
$$

Now, either we have two complex conjugate roots (we shall refer to this situation as the " $\beta$-case") or we have two real roots (which we shall refer to as the "s-case") of which one root must be -1 in order for the state to evolve to an orthogonal state.

Let us now consider each of these possibilities in detail.

1. $\beta$-case

Assume that $e^{i \beta}, e^{-i \beta}$ are the complex roots of (24), so that

$$
\begin{aligned}
P(x) & =\left[\left|a_{2}\right|^{2}+\left|a_{3}\right|^{2}\right]\left\{x^{2}+\frac{\left|a_{1}\right|^{2}}{\left|a_{2}\right|^{2}+\left|a_{3}\right|^{2}} x+\frac{\left|a_{0}\right|^{2}}{\left|a_{2}\right|^{2}+\left|a_{3}\right|^{2}}\right\} \\
& =\left[\left|a_{2}\right|^{2}+\left|a_{3}\right|^{2}\right]\left\{x-e^{i \beta}\right\}\left\{x-e^{-i \beta}\right\} \\
& =\left[\left|a_{2}\right|^{2}+\left|a_{3}\right|^{2}\right]\left\{x^{2}-2 \cos \beta x+1\right\} .
\end{aligned}
$$

Comparing eqs. (25) and (27) yields

$$
\frac{\left|a_{1}\right|^{2}}{\left|a_{2}\right|^{2}+\left|a_{3}\right|^{2}}=-2 \cos \beta, \quad \frac{\left|a_{0}\right|^{2}}{\left|a_{2}\right|^{2}+\left|a_{3}\right|^{2}}=1 .
$$

This, together with the normalization condition, gives

$$
\begin{aligned}
\left|a_{0}\right|^{2} & =\frac{1}{2(1-\cos \beta)}, \\
\left|a_{1}\right|^{2} & =\frac{-\cos \beta}{1-\cos \beta}, \\
\left|a_{2}\right|^{2} & =\frac{\delta}{2(1-\cos \beta)}, \\
\left|a_{3}\right|^{2} & =\frac{1-\delta}{2(1-\cos \beta)} .
\end{aligned}
$$

Since the squares of the absolute values of the coefficients have to be positive, $\beta$ is restricted to the interval $\left[\frac{\pi}{2}, \pi\right]$ and $\delta \in[0,1]$. Introducing the phases $0 \leq \phi_{1}, \phi_{2}, \phi_{3}<2 \pi$, we have 


$$
\begin{aligned}
& a_{0}=\frac{1}{\sqrt{2(1-\cos \beta)}}, \\
& a_{1}=e^{i \phi_{1}} \sqrt{\frac{-\cos \beta}{1-\cos \beta}}, \\
& a_{2}=e^{i \phi_{2}} \sqrt{\frac{\delta}{2(1-\cos \beta)}}, \\
& a_{3}=e^{i \phi_{3}} \sqrt{\frac{1-\delta}{2(1-\cos \beta)}} .
\end{aligned}
$$

The expression for the time dependent entanglement in terms of the above parameters is

$$
\begin{aligned}
\mathcal{E}\left(\beta, \delta, \phi_{1}, \phi_{2}, \phi_{3}, \omega_{0} t\right)= & \frac{1}{4(\cos \beta-1)^{2}}\left(1-8 \cos \beta-4 \sqrt{-2 \cos \beta}\left(\delta \cos \left(12 \omega_{0} t+\phi_{1}-2 \phi_{2}\right)\right.\right. \\
& \left.\left.+(\delta-1) \cos \left(12 \omega_{0} t+\phi_{1}-2 \phi_{3}\right)\right)+4 \delta(\delta-1) \cos ^{2}\left(\phi_{2}-\phi_{3}\right)\right) .
\end{aligned}
$$

The time needed to reach an orthogonal state is

$$
\tau(\beta)=\frac{\beta}{4 \omega_{0}},
$$

the mean energy is $E=4 \hbar \omega_{0}$ and the dispersion is $\Delta E=\frac{4 \hbar \omega_{0}}{\sqrt{1-\cos \beta}}$. In this case the dispersion of the energy is always less than the mean energy and thus we have for the minimum possible time

$$
\tau_{\min }(\beta)=\frac{\pi \sqrt{1-\cos \beta}}{8 \omega_{0}} .
$$

The quantities we are interested in are

$$
\frac{\tau}{\tau_{\min }}(\beta)=\frac{2 \beta}{\pi \sqrt{1-\cos \beta}},
$$

and the entanglement $\mathcal{E}$ associated with the $\beta$-case states.

Fig.1 depicts the region in the $\left(\mathcal{E}, \frac{\tau}{\tau_{\min }}\right)$-plane corresponding to the family of initial states comprising the $\beta$-case. It can be verified after some algebra that this region coincides with the region associated with initial states belonging to the $\beta$-case and having vanishing phases $\left\{\phi_{1}, \phi_{2}, \phi_{3}\right\}$. The entanglement of these states is given by, 


$$
\mathcal{E}(\beta, \delta)=\frac{(1-2 \delta)(1-2 \delta+4 \sqrt{2} \sqrt{-\cos \beta})-8 \cos \beta}{4(-1+\cos \beta)^{2}} .
$$

The boundary curves of the allowed region depicted in Fig.1 starting at the top and moving counterclockwise, are given by

$$
\begin{aligned}
& \text { A } \beta=\pi, \quad \delta \in[0,1], \\
& \quad\left\{\frac{1}{16}(9-4 \sqrt{2}) \leq \mathcal{E} \leq \frac{1}{16}(9+4 \sqrt{2}), \tau / \tau_{\min }=\sqrt{2}\right\}, \\
& \text { B } \beta \in[\arccos (-1 / 8), \pi], \quad \delta=1, \\
& \quad\left\{0 \leq \mathcal{E} \leq \frac{1}{16}(9-4 \sqrt{2}), \frac{4 \sqrt{2} \arccos (-1 / 8)}{3 \pi} \leq \tau / \tau_{\min } \leq \sqrt{2}\right\}, \\
& \text { C } \beta \in\left[\frac{\pi}{2}, \arccos (-1 / 8)\right], \quad \delta=\frac{1}{2}(1+2 \sqrt{-\csc (\beta) \sin (2 \beta)}) \\
& \quad\left\{\mathcal{E}=0,1 \leq \tau / \tau_{\min } \leq \frac{4 \sqrt{2} \arccos (-1 / 8)}{3 \pi}\right\}, \\
& \text { D } \beta=\frac{\pi}{2}, \quad \delta \in[0,1], \\
& \quad\left\{0 \leq \mathcal{E} \leq \frac{1}{4}, \tau / \tau_{\min }=1\right\}, \\
& \text { E } \beta \in\left[\frac{\pi}{2}, \pi\right], \quad \delta=0, \\
& \quad\left\{\frac{1}{4} \leq \mathcal{E} \leq \frac{1}{16}(9+4 \sqrt{2}), 1 \leq \tau / \tau_{\min } \leq \sqrt{2}\right\} .
\end{aligned}
$$

We can see in Fig. 1 that the initial states within the $\beta$-case that evolve into an orthogonal state cover a large part of the $\left(\mathcal{E}, \frac{\tau}{\tau_{\min }}\right)$-plane. There are, however, some relevant features of Fig.1 pointing towards an interesting connection between entanglement and quantum speed. First, we see that only states of relatively low entanglement (more precisely, states with entanglement values within the interval $0 \leq \mathcal{E} \leq \frac{1}{4}$ ) achieve the quantum speed limit given by $\frac{\tau}{\tau_{\min }}=1$. Moreover, separable states (that is, those with $\mathcal{E}=0$ ) that reach an orthogonal state have orthogonality times $\tau$ quite close to the minimum one $\tau_{\min }$. Indeed, the separable states represented in Fig. 1 satisfy $1 \leq \frac{\tau}{\tau_{\min }} \leq \frac{4 \sqrt{2} \arccos (-1 / 8)}{3 \pi}$. It also transpires from Fig.1 that there are no slow states (that is, having large values of $\frac{\tau}{\tau_{\min }}$ ) with low entanglement.

Let us now consider the time averaged entanglement

$$
\langle\mathcal{E}\rangle=\frac{1}{\tau} \int_{0}^{\tau} \mathcal{E} d t
$$

of initial states corresponding to the $\beta$-case. The expression for the averaged entanglement is given by 


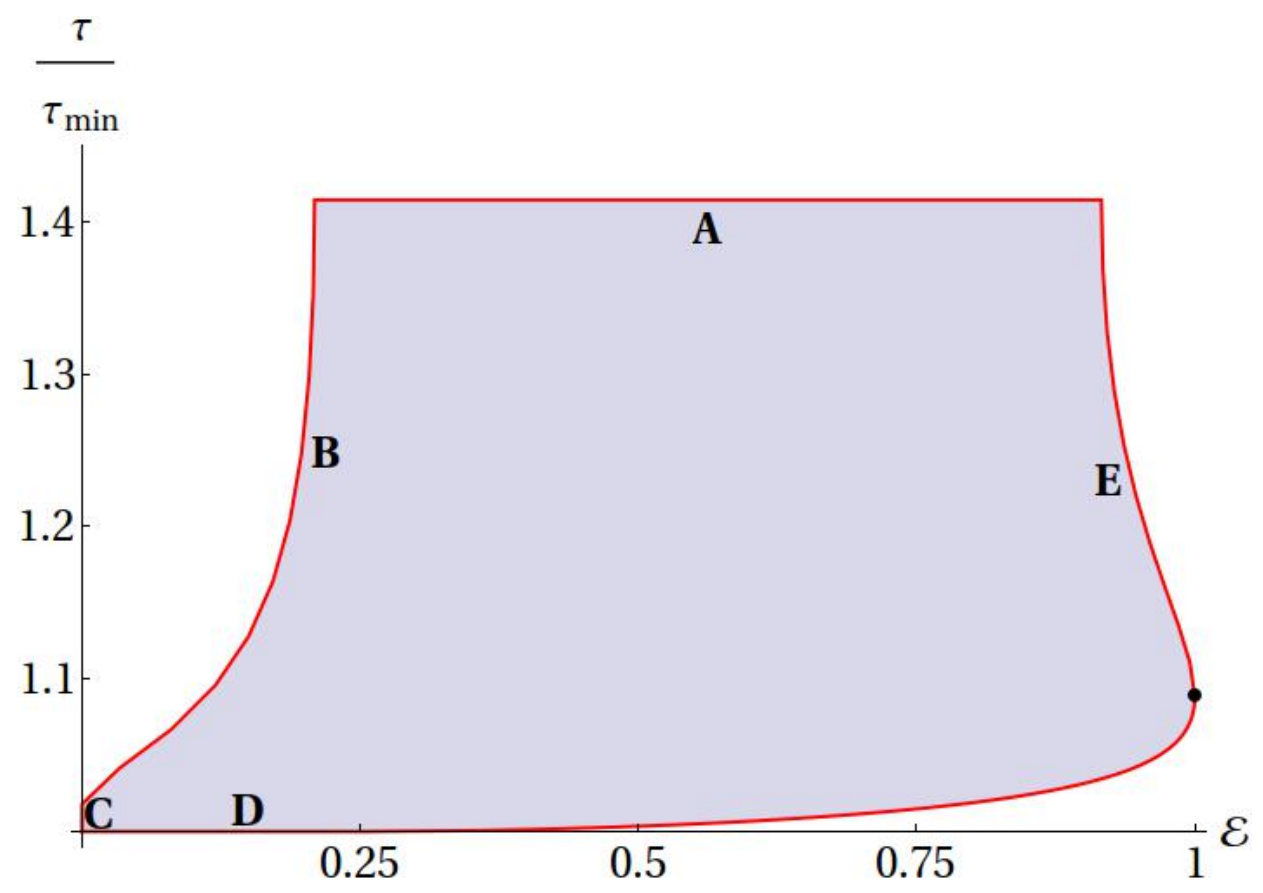

FIG. 1: Initial states corresponding to the $\beta$-case. The dot on the boundary curve corresponds to the initial states of highest entanglement that evolve into an orthogonal state. These states have $\mathcal{E}=1$ and $\frac{\tau}{\tau_{\min }}=\frac{4}{3} \sqrt{\frac{2}{3}}$. All depicted quantities are dimensionless.

$$
\begin{aligned}
\left\langle\mathcal{E}\left(\beta, \delta, \phi_{1}, \phi_{2}, \phi_{3}\right)\right\rangle= & \frac{\csc \left(\frac{\beta}{2}\right)^{4}}{48 \beta}\left(6 \beta \delta(\delta-1) \cos ^{2}\left(\phi_{2}-\phi_{3}\right)+\right. \\
& +3 \beta(1-8 \cos (\beta))-8 \sqrt{-2 \cos (\beta)} \sin \left(\frac{3 \beta}{2}\right)\left(\delta \cos \left(\frac{3 \beta}{2}+\phi_{1}-2 \phi_{2}\right)+\right. \\
& \left.\left.+(\delta-1) \cos \left(\frac{3 \beta}{2}+\phi_{1}-2 \phi_{3}\right)\right)\right) .
\end{aligned}
$$

We can see in Fig.2 the region in the $\left(\langle\mathcal{E}\rangle, \frac{\tau}{\tau_{\min }}\right)$-plane associated with initial states corresponding to the $\beta$-case. The boundary lines of this region are given by,
A $\beta=\pi, \quad \delta \in[0.5,1]$,
$\left\{\frac{1}{2} \leq\langle\mathcal{E}\rangle \leq \frac{9}{16}, \tau / \tau_{\min }=\sqrt{2}\right\}$,
B $\beta \in\left[\frac{\pi}{2}, \pi\right], \quad \delta=\frac{3 \beta+2 \sin (3 \beta) \sqrt{-\csc (\beta) \sin (2 \beta)}}{6 \beta}$, $\left\{0 \leq\langle\mathcal{E}\rangle \leq \frac{1}{2}, 0 \leq \tau / \tau_{\min } \leq \sqrt{2}\right\}$, 


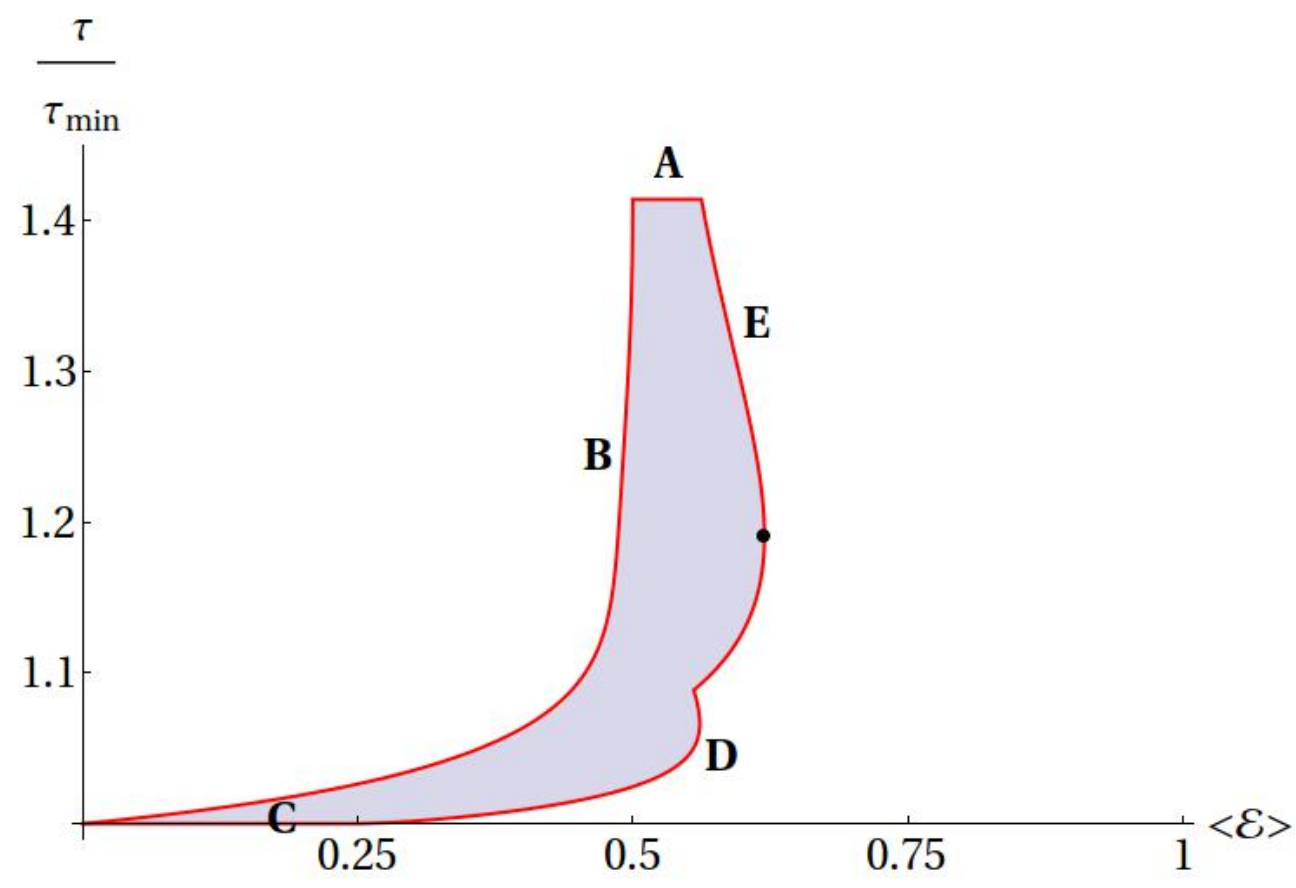

FIG. 2: Initial states corresponding to the $\beta$-case, with time averaged entanglement. The dot on the boundary curve corresponds to the initial states leading to the highest time averaged entanglement. These states have $\langle\mathcal{E}\rangle \approx 0.62$ and $\frac{\tau}{\tau_{\min }} \approx 1.19$. All depicted quantities are dimensionless.

$$
\begin{aligned}
& \text { C } \beta=\frac{\pi}{2}, \quad \delta \in[0.5,1], \\
& \left\{0 \leq\langle\mathcal{E}\rangle \leq \frac{1}{4}, \tau / \tau_{\min }=0,\right. \\
& \text { D } \beta \in\left[\frac{\pi}{2}, \frac{2 \pi}{3}\right], \quad \delta=1, \\
& \quad\left\{0 \leq\langle\mathcal{E}\rangle \leq \frac{5}{9}, \quad 1 \leq \tau / \tau_{\min } \leq \frac{4}{3} \sqrt{\frac{2}{3}}\right\}, \\
& \text { E } \beta \in\left[\frac{2 \pi}{3}, \pi\right], \quad \delta=0, \\
& \quad\left\{\frac{5}{9} \leq\langle\mathcal{E}\rangle \leq \frac{9}{16}, \frac{4}{3} \sqrt{\frac{2}{3}} \leq \tau / \tau_{\min } \leq \sqrt{2}\right\} .
\end{aligned}
$$

The connection between entanglement and speed of quantum evolution for the $\beta$-case states can be appreciated more clearly when plotting these states in the $\left(\langle\mathcal{E}\rangle, \frac{\tau}{\tau_{\min }}\right)$-plane than when plotting them in the $\left(\mathcal{E}, \frac{\tau}{\tau_{\min }}\right)$-plane. We observe in Fig.2 that all initial states that reach the speed limit give rise to time evolutions with low average entanglement in the range $0 \leq\langle\mathcal{E}\rangle \leq \frac{1}{4}$. Actually, all initial states that generate evolutions with low average entanglement (in the abovementioned range) are endowed with orthogonality times close to the minimum one $\tau_{\text {min }}$. In other words, within the $\beta$-case states there are no slow states 
of low entanglement, as attested by the large upper-left empty region in Fig.2. Slow states characterized by large values of the ratio $\frac{\tau}{\tau_{\min }}$ are concentrated in the region of medium time averaged entanglement values. There are no states in Fig.2 with large values of $\langle\mathcal{E}\rangle$, meaning that states leading to high values of the time averaged entanglement do not reach an orthogonal state at all. Finally, note that Figures 1 and 2 have the same range of values of $\frac{\tau}{\tau_{\min }}$ (and, in particular, the same maximum value of this quantity). This is due to the fact that $\frac{\tau}{\tau_{\min }}$ is determined solely by the initial state, and does not depend on which particular measure we use to characterize the amount of entanglement involved during the evolution of the system under consideration.

Symmetric states are of special interest when investigating the connection between entanglement and quantum speed, since they correspond to the most efficient use of the available energy resources. In the case of symmetric states entanglement is strictly necessary in order to reach the quantum speed limit, that is, in order to have the minimum orthogonality time $\tau=\tau_{\text {min }}$. On the contrary, there are some particular highly asymmetric states, where all the energetic resources are concentrated in one of the qubits, that achieve the speed limit without any entanglement. These are separable states of non-interacting qubits where only one of the qubits actually evolves and the remaining qubits are in eigenstates of their corresponding Hamiltonians. This is a marginal case where, from the dynamical point of view, one effectively deals with a single-qubit system, and the composite character of the whole system plays no physical role (see [18] and references therein).

For a symmetric state the coefficients of the states $|01\rangle$ and $|10\rangle$ in eq.(12) must be equal. This only holds when $a_{3}=0$ which, in turn, corresponds to $\delta=1$. The coefficients associated with symmetric states are then of the form

$$
\begin{aligned}
& a_{0}=\frac{1}{\sqrt{2(1-\cos \beta)}}, \\
& a_{1}=e^{i \phi_{1}} \sqrt{\frac{-\cos \beta}{1-\cos \beta}}, \\
& a_{2}=e^{i \phi_{2}} \frac{1}{\sqrt{2(1-\cos \beta)}}, \\
& a_{3}=0 .
\end{aligned}
$$

with $\beta \in\left[\frac{\pi}{2}, \pi\right]$.

Figure 3 shows the region in the $\left(\mathcal{E}, \tau / \tau_{\text {min }}\right)$-plane which the symmetric $\beta$-case states 
occupy. The boundaries of this region are given by

$$
\begin{aligned}
& \text { A } \beta=\pi, \quad \phi_{1}=\phi_{2} \in[0, \pi], \\
& \quad\left\{\frac{1}{16}(9-4 \sqrt{2}) \leq \mathcal{E} \leq \frac{1}{16}(9+4 \sqrt{2}), \tau / \tau_{\min }=\sqrt{2}\right\}, \\
& \text { B } \beta \in[\arccos (-1 / 8), \pi], \quad \phi_{1}=\phi_{2}=0, \\
& \quad\left\{0 \leq \mathcal{E} \leq \frac{1}{16}(9-4 \sqrt{2}), \frac{4 \sqrt{2} \arccos (-1 / 8)}{3 \pi} \leq \tau / \tau_{\min } \leq \sqrt{2}\right\}, \\
& \text { C } \beta \in[\pi / 2, \arccos (-1 / 8)], \quad \phi_{1}=\phi_{2}=0, \\
& \quad\left\{0 \leq \mathcal{E} \leq \frac{1}{4}, 1 \leq \tau / \tau_{\min } \leq \frac{4 \sqrt{2} \arccos (-1 / 8)}{3 \pi}\right\}, \\
& \text { D } \beta \in\left[\frac{\pi}{2}, \pi\right], \quad \phi_{1}=\phi_{2}=\pi, \\
& \quad\left\{\frac{1}{4} \leq \mathcal{E} \leq \frac{1}{16}(9+4 \sqrt{2}), 1 \leq \tau / \tau_{\min } \leq \sqrt{2}\right\} .
\end{aligned}
$$

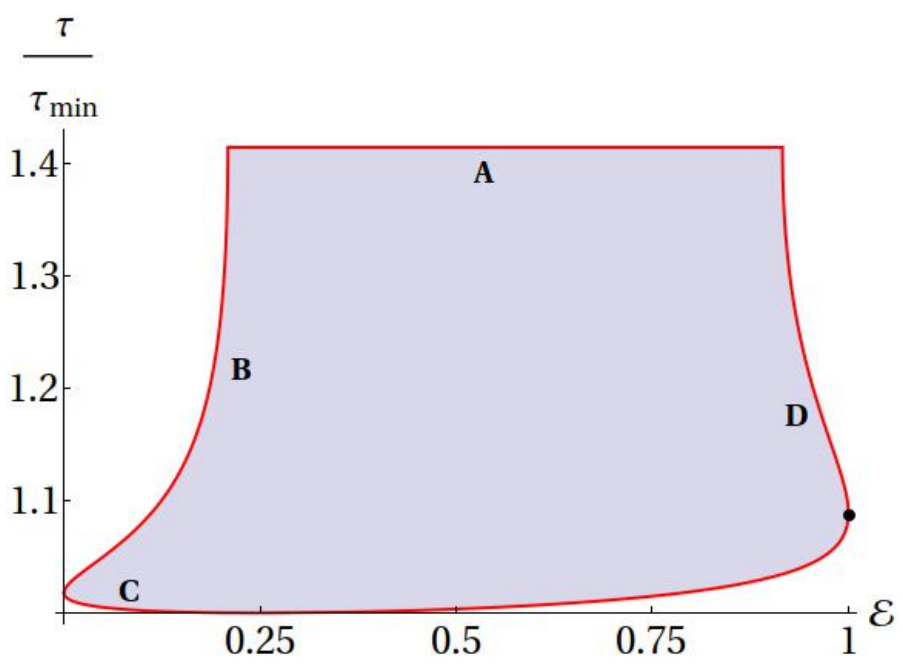

FIG. 3: $\mathcal{E}$ vs $\tau / \tau_{\min }$ for the symmetric initial states belonging to the $\beta$-case. The dot on the boundary curve corresponds to the initial symmetric states of highest entanglement that evolve into an orthogonal state. All depicted quantities are dimensionless.

The general features of Fig.3 are similar to those of Fig.1. There is, however, an interesting difference: the only symmetric $\beta$-case states that achieve the speed limit have one particular entanglement value, $\mathcal{E}=\frac{1}{4}$.

In Fig.4 the symmetric $\beta$-case states are represented in the $\left(\langle\mathcal{E}\rangle, \frac{\tau}{\tau_{\min }}\right)$-plane. The boundaries of this region are given by,
A $\beta=\pi, \quad \phi_{1} \in[0, \pi], \quad \phi_{2}=\frac{5 \pi}{4}$,
$\left\{\frac{9}{16}-\frac{1}{3 \sqrt{2} \pi} \leq\langle\mathcal{E}\rangle \leq \frac{9}{16}+\frac{1}{3 \sqrt{2} \pi}, \tau / \tau_{\min }=\sqrt{2}\right\}$, 


$$
\begin{aligned}
& \text { B } \beta \in\left[\frac{2 \pi}{3}, \pi\right], \quad \phi_{1}=0, \quad \phi_{2}=\frac{3 \beta+2 \pi}{4}, \\
& \quad\left\{\frac{9}{16}-\frac{1}{3 \sqrt{2} \pi} \leq\langle\mathcal{E}\rangle \leq \frac{5}{9}, \quad \frac{4}{3} \sqrt{\frac{2}{3}} \leq \tau / \tau_{\min } \leq \sqrt{2}\right\}, \\
& \text { C } \beta \in\left[1.5861, \frac{2 \pi}{3}\right], \quad \phi_{1}=\pi, \quad \phi_{2}=\frac{3 \beta+2 \pi}{4}, \\
& \quad\left\{0.2229 \leq\langle\mathcal{E}\rangle \leq \frac{5}{9}, 1.0021 \leq \tau / \tau_{\min } \leq \frac{4}{3} \sqrt{\frac{2}{3}}\right\}, \\
& \text { D } \beta \in\left[\frac{\pi}{2}, 1.5861\right], \quad \phi_{1}=\pi, \quad \phi_{2}=\frac{3 \beta+2 \pi}{4}, \\
& \quad\left\{0.2229 \leq\langle\mathcal{E}\rangle \leq \frac{1}{4}, 1 \leq \tau / \tau_{\min } \leq 1.0021\right\}, \\
& \text { E } \beta \in\left[\frac{\pi}{2}, \frac{2 \pi}{3}\right], \quad \phi_{1}=0, \quad \phi_{2}=\frac{3 \beta+2 \pi}{4}, \\
& \quad\left\{\frac{1}{4} \leq\langle\mathcal{E}\rangle \leq \frac{5}{9}, 1 \leq \tau / \tau_{\min } \leq \frac{4}{3} \sqrt{\frac{2}{3}}\right\}, \\
& \text { F } \beta \in\left[\frac{2 \pi}{3}, \pi\right], \quad \phi_{1}=\pi, \quad \phi_{2}=\frac{3 \beta+2 \pi}{4}, \\
& \quad\left\{\frac{5}{9} \leq\langle\mathcal{E}\rangle \leq \frac{9}{16}+\frac{1}{3 \sqrt{2} \pi}, \quad \frac{4}{3} \sqrt{\frac{2}{3}} \leq \tau / \tau_{\min } \leq \sqrt{2}\right\} .
\end{aligned}
$$

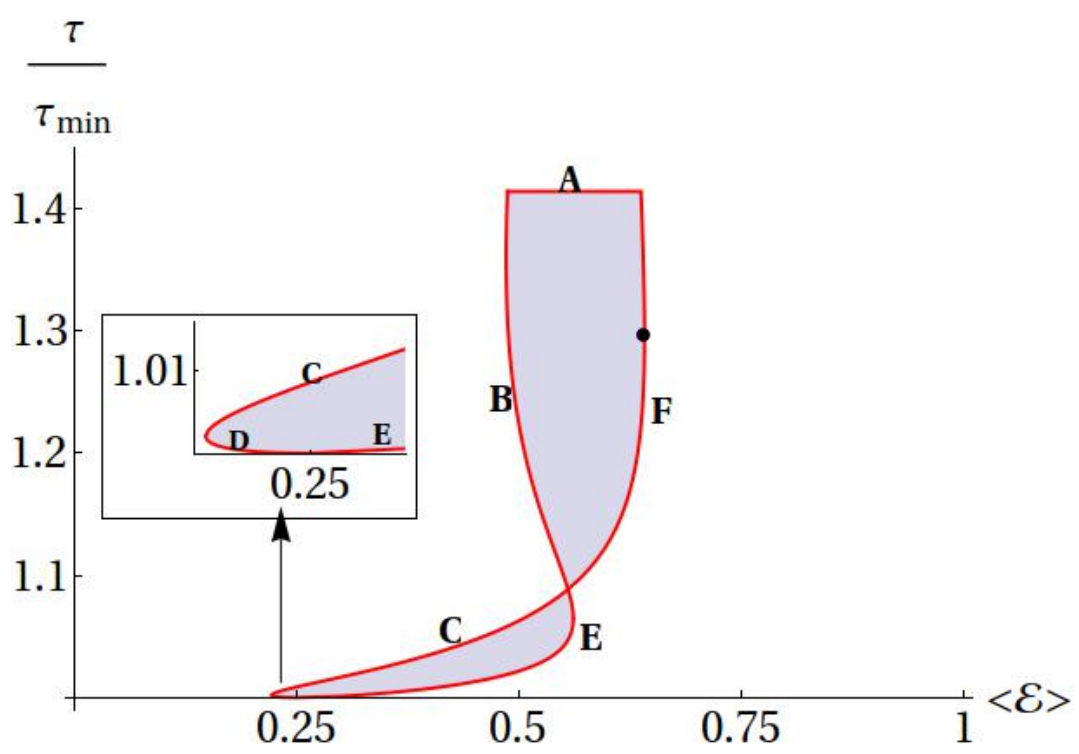

FIG. 4: Symmetric initial states corresponding to the $\beta$-case, with time averaged entanglement. The dot on the boundary curve corresponds to the initial symmetric states that evolve into an orthogonal state with the highest time averaged entanglement. All depicted quantities are dimensionless.

It can be appreciated in Fig. 4 that there is a clear tendency for the quotient $\frac{\tau}{\tau_{\min }}$ to increase as the time averaged entanglement $\langle\mathcal{E}\rangle$ increases: that is, lower entanglement tends to enhance the speed of quantum evolution. 
2. $s$-case

One of the real roots has to be -1 and let the other one be $s$. Then we have

$$
\begin{aligned}
P(x) & =\left[\left|a_{2}\right|^{2}+\left|a_{3}\right|^{2}\right]\left\{x^{2}+\frac{\left|a_{1}\right|^{2}}{\left|a_{2}\right|^{2}+\left|a_{3}\right|^{2}} x+\frac{\left|a_{0}\right|^{2}}{\left|a_{2}\right|^{2}+\left|a_{3}\right|^{2}}\right\} \\
& =\left[\left|a_{2}\right|^{2}+\left|a_{3}\right|^{2}\right]\left\{x^{2}+(1-s) x-s\right\} .
\end{aligned}
$$

Comparing eqs. (39) and (40) gives

$$
\frac{\left|a_{1}\right|^{2}}{\left|a_{2}\right|^{2}+\left|a_{3}\right|^{2}}=1-s, \quad \frac{\left|a_{0}\right|^{2}}{\left|a_{2}\right|^{2}+\left|a_{3}\right|^{2}}=-s .
$$

This, together with the normalization condition, gives

$$
\begin{aligned}
\left|a_{0}\right|^{2} & =\frac{-s}{2(1-s)}, \\
\left|a_{1}\right|^{2} & =\frac{1-s}{2(1-s)}=\frac{1}{2}, \\
\left|a_{2}\right|^{2} & =\frac{\lambda}{2(1-s)}, \\
\left|a_{3}\right|^{2} & =\frac{1-\lambda}{2(1-s)},
\end{aligned}
$$

with $\lambda \in[0,1]$. From the positivity condition we see that $s \leq 0$. Again introducing three phases $\mu_{1}, \mu_{2}, \mu_{3} \in[0,2 \pi)$ we have

$$
\begin{aligned}
& a_{0}=\sqrt{\frac{-s}{2(1-s)}}, \\
& a_{1}=e^{i \mu_{1}} \frac{1}{\sqrt{2}}, \\
& a_{2}=e^{i \mu_{2}} \sqrt{\frac{\lambda}{2(1-s)}}, \\
& a_{3}=e^{i \mu_{3}} \sqrt{\frac{1-\lambda}{2(1-s)}} .
\end{aligned}
$$

The linear entanglement is hence 


$$
\begin{aligned}
\mathcal{E}\left(s, \lambda, \mu_{1}, \mu_{2}, \mu_{3}, \omega_{0} t\right)= & \frac{1}{4(1-s)^{5 / 2}}\left(4 ( s - 1 ) \sqrt { - s } \left(\lambda \cos \left(12 \omega_{0} t+\mu_{1}-2 \mu_{2}\right)+\right.\right. \\
& \left.+(\lambda-1) \cos \left(12 \omega_{0} t+\mu_{1}-2 \mu_{3}\right)\right)+ \\
& \left.+\sqrt{1-s}\left(1+4 s(s-1)+4 \lambda(\lambda-1) \cos ^{2}\left(\mu_{2}-\mu_{3}\right)\right)\right) .
\end{aligned}
$$

Since one of the roots is equal to -1 , the time needed to reach an orthogonal state is constant, implying that all the states that do evolve to an orthogonal state take the same amount of time, namely

$$
\tau=\frac{\pi}{4 \omega_{0}} .
$$

What differs among the states are the energy resources:

$$
E=\frac{2 \omega_{0} \hbar(3-s)}{1-s}, \quad \Delta E=\frac{2 \omega_{0} \hbar \sqrt{1-6 s+s^{2}}}{1-s}
$$

This results in

$$
\tau_{\min }=\frac{\pi(1-s)}{4 \omega_{0} \sqrt{1-6 s+s^{2}}}
$$

and

$$
\frac{\tau}{\tau_{\min }}(s)=\frac{\sqrt{1-6 s+s^{2}}}{1-s} .
$$

In Fig. 5 we can see the region in the $\left(\mathcal{E}, \tau / \tau_{\min }\right)$-plane corresponding to the $s$-case. It can be verified that the boundaries of this region coincide with those corresponding to $s$ case initial states with the phases $\mu_{1}, \mu_{2}, \mu_{3}$ all equal to zero. The linear entanglement then becomes

$$
\mathcal{E}(s, \lambda)=\frac{4(-1+s) \sqrt{-s}(-1+2 \lambda)+\sqrt{1-s}(1+4(-1+s) s+4(-1+\lambda) \lambda)}{4(1-s)^{5 / 2}} .
$$

The boundary curves of the region depicted in Fig. 5 are given by

$$
\begin{aligned}
\text { A } & s=-1, \quad \lambda \in[0,1], \\
& \left\{\frac{1}{16}(9-4 \sqrt{2}) \leq \mathcal{E} \leq \frac{1}{16}(9+4 \sqrt{2}), \quad \tau / \tau_{\min }=\sqrt{2}\right\},
\end{aligned}
$$




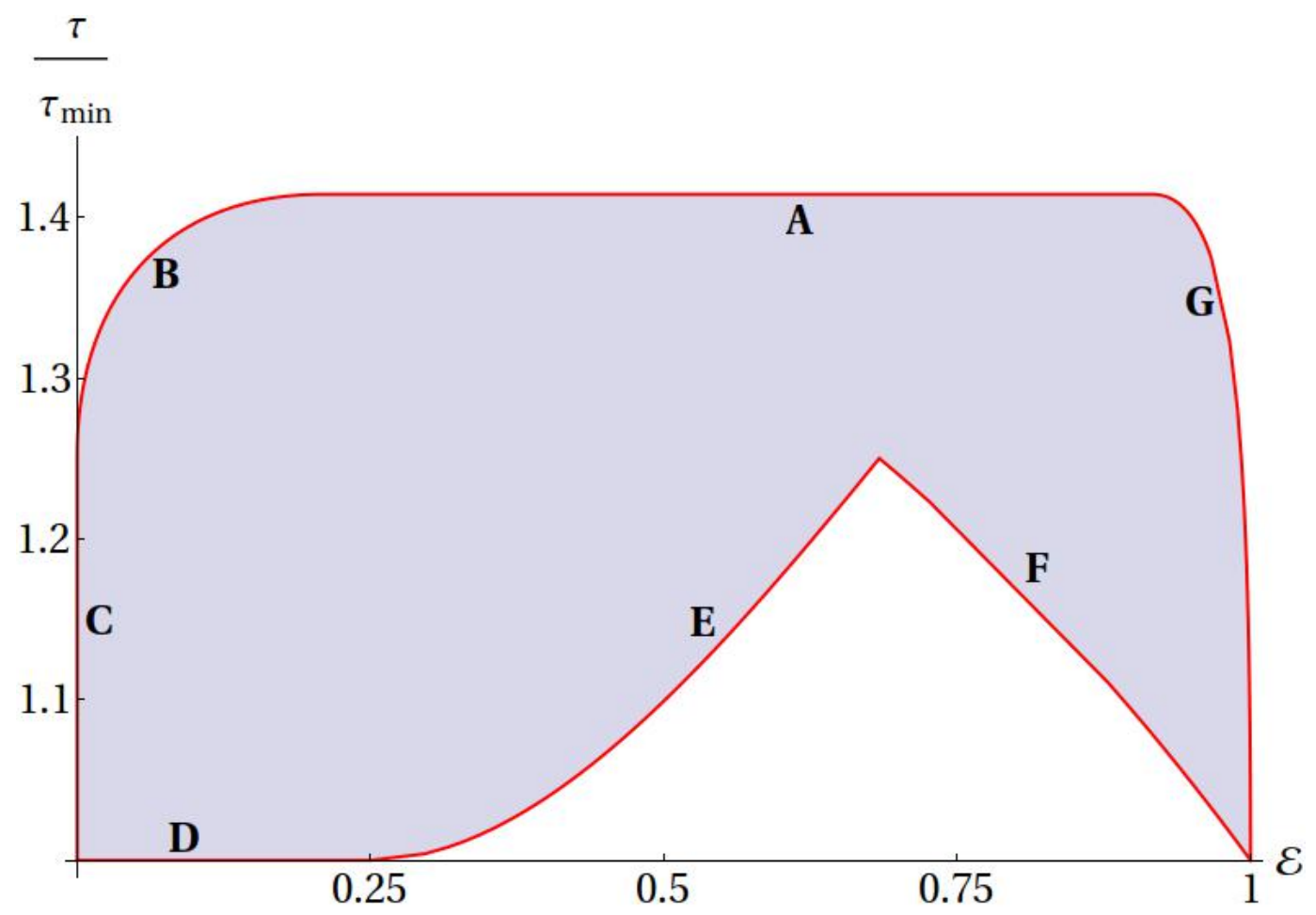

FIG. 5: Initial states corresponding to the $s$-case. All depicted quantities are dimensionless.

$$
\begin{aligned}
\text { B } & s \in\left[-1, \frac{1-\sqrt{2}}{2}\right], \quad \lambda=1, \\
& \left\{0 \leq \mathcal{E} \leq \frac{1}{16}(9-4 \sqrt{2}), \quad \tau / \tau_{\min } \in[1, \sqrt{40 \sqrt{2}-55}\},\right. \\
\text { C } & s \in\left[\frac{1-\sqrt{2}}{2}, 0\right], \quad \lambda=\frac{1}{2} \sqrt{s(s-1)}, \\
& \left\{\mathcal{E}=0, \quad \tau / \tau_{\min } \in[1, \sqrt{40 \sqrt{2}-55}]\right\}, \\
\text { D } & s=0, \quad \lambda \in[0,1], \\
& \left\{0 \leq \mathcal{E} \leq \frac{1}{4}, \quad \tau / \tau_{\min }=1\right\}, \\
\text { E } & s \in\left[0, \frac{1}{9}(-23+8 \sqrt{7})\right], \quad \lambda=0, \\
& \left\{0 \leq \mathcal{E} \leq \frac{175}{256}, \quad 1 \leq \tau / \tau_{\min } \leq \frac{5}{4}\right\}, \\
\text { F } & s \in\left(-\infty, \frac{1}{9}(-23-8 \sqrt{7})\right], \quad \lambda=1, \\
& \left\{\frac{175}{256} \leq \mathcal{E} \leq 1, \quad \frac{5}{4} \leq \tau / \tau_{\min } \leq 1\right\}, \\
\text { G } & s \in(-\infty,-1], \quad \lambda=0, \\
& \left\{\frac{1}{16}(9+4 \sqrt{2}) \leq \mathcal{E} \leq 1, \quad 1 \leq \tau / \tau_{\min } \leq \sqrt{2}\right\} .
\end{aligned}
$$


Fig.6 corresponds to $s$-case initial states with time averaged entanglement, which can be computed using the following expression,

$$
\begin{aligned}
<\mathcal{E}\left(s, \lambda, \mu_{1}, \mu_{2}, \mu_{3}\right)>= & \frac{1}{12 \pi(1-s)^{5 / 2}}\left(8 ( 1 - s ) \sqrt { - s } \left(\lambda \sin \left(\mu_{1}-2 \mu_{2}\right)+\right.\right. \\
& \left.+(\lambda-1) \sin \left(\mu_{1}-2 \mu_{3}\right)\right)+ \\
& \left.+3 \pi \sqrt{1-s}\left(1+4 s(s-1)+4 \lambda(\lambda-1) \cos ^{2}\left(\mu_{2}-\mu_{3}\right)\right)\right) .
\end{aligned}
$$

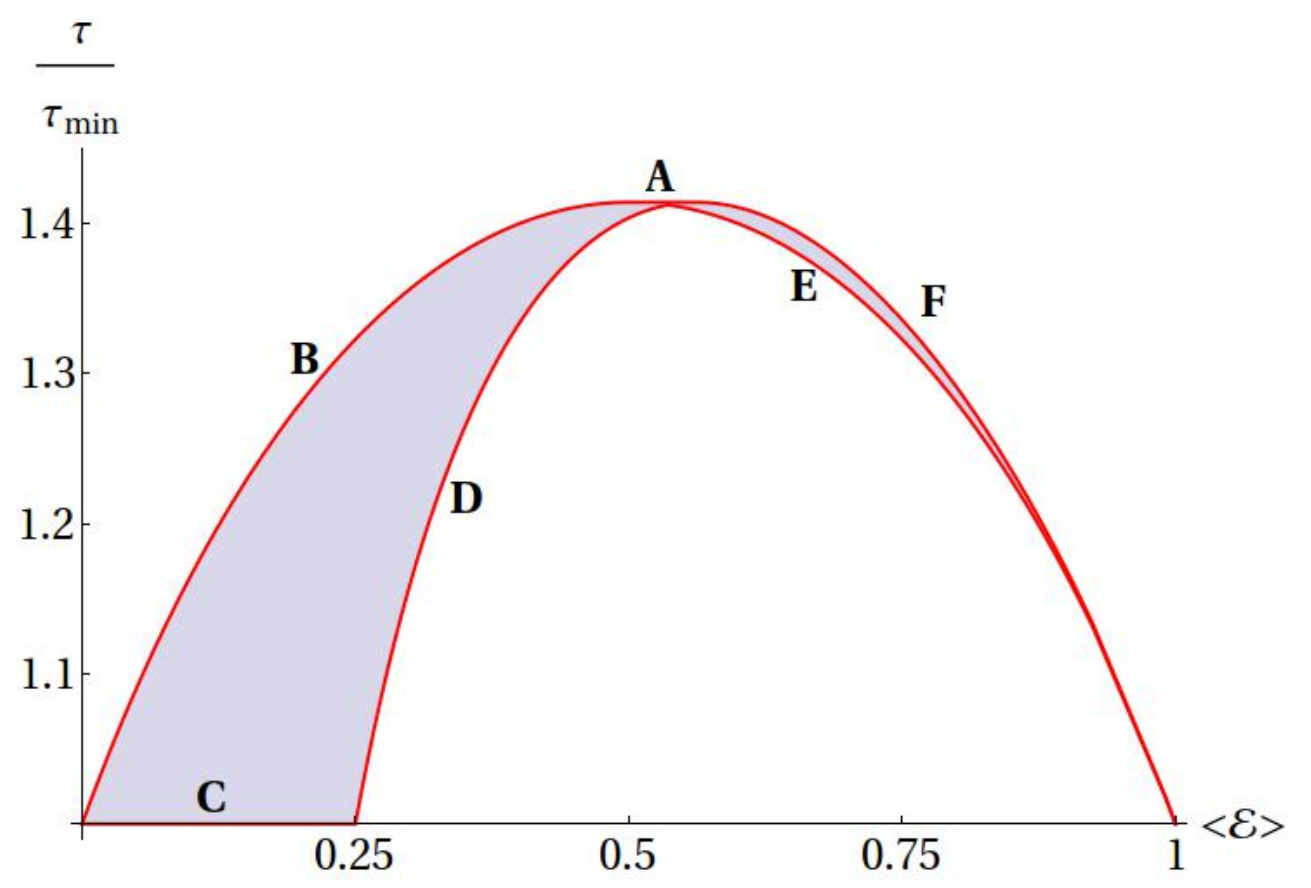

FIG. 6: Initial states corresponding to the $s$-case, with time averaged entanglement. All depicted quantities are dimensionless.

The boundaries of the allowed region depicted in Fig.6 are given by,
A $s=-1, \quad \lambda \in[0.5,1]$,
$\left\{\frac{1}{2} \leq\langle\mathcal{E}\rangle \leq \frac{9}{16}, \quad \tau / \tau_{\min }=\sqrt{2}\right\}$,
B $s \in[-1,0], \quad \lambda=0.5$,
$\left\{0 \leq\langle\mathcal{E}\rangle \leq \frac{1}{2}, \quad \tau / \tau_{\min } \in[1, \sqrt{2}\}\right.$, 


$$
\begin{array}{rl}
\mathbf{C} & s=0, \quad \lambda \in[0.5,1], \\
& \left\{0 \leq\langle\mathcal{E}\rangle \leq \frac{1}{4}, \quad \tau / \tau_{\min }=1\right\}, \\
\mathbf{D} & s \in\left[-\frac{\sqrt{3}}{2}, 0\right], \quad \lambda=0, \\
& \left\{1 / 4 \leq\langle\mathcal{E}\rangle \leq 4-2 \sqrt{3}, \quad 1 \leq \tau / \tau_{\min } \leq \sqrt{56 \sqrt{3}-95}\right\}, \\
\mathbf{E} & s \in\left(-\infty,-\frac{2}{\sqrt{3}}\right], \quad \lambda=0.5, \\
& \left\{4-2 \sqrt{3} \leq\langle\mathcal{E}\rangle \leq 1, \quad 1 \leq \tau / \tau_{\min } \leq \sqrt{56 \sqrt{3}-95}\right\}, \\
& \mathbf{F} \quad s \in[-1,-\infty), \quad \lambda=1, \\
& \left\{\frac{9}{16} \leq\langle\mathcal{E}\rangle \leq 1, \quad 1 \leq \tau / \tau_{\min } \leq \sqrt{2}\right\} .
\end{array}
$$

To find the states evolving at the speed limit, we set $\tau / \tau_{\min }(s)=1$. This means either $s=0$ or $s \rightarrow-\infty$. The former instance yields $0 \leq \mathcal{E} \leq \frac{1}{4}$, whereas the latter gives $\mathcal{E}=1$. The symmetric states correspond to $\lambda=1$ and thus have $\mathcal{E}=\frac{1}{4}$. In contrast to what happens in the $\beta$-case, we can see in Figures 5 and 6 that there are maximally entangled states that reach the speed limit. These states evolve in a time-optimal way in the sense of having $\frac{\tau}{\tau_{\min }}=1$. However, in terms of the absolute orthogonality time $\tau$, the $s$-case states are slow. Actually, they are the slowest states, because they have the largest possible orthogonality time, given by $\tau=\frac{\pi}{4 \omega_{0}}$.

\section{MORE GENERAL VALUES OF $\omega$ AND $\omega_{0}$ : SEMI-NUMERICAL TREAT- MENT}

We want to find solutions to the equation

$$
\left\langle\psi\left(t_{0}\right) \mid \psi\left(t_{0}+\tau\right)\right\rangle^{*}=\left|a_{0}\right|^{2}+e^{4 i \omega_{0} \tau}\left|a_{1}\right|^{2}+e^{2 i\left(\omega+\omega_{0}\right) \tau}\left[\left|a_{2}\right|^{2}+\left|a_{3}\right|^{2}\right]=0 .
$$

By equating the real and imaginary parts to zero, one obtains two equations which, together with the normalization condition, constitute a set of three linear equations in $\left|a_{0}\right|^{2},\left|a_{1}\right|^{2}$, and $\left|a_{2}\right|^{2}+\left|a_{3}\right|^{2}$. These equations lead to

$$
\left|a_{0}\right|^{2}=\frac{\sin \left[2\left(\omega-\omega_{0}\right) \tau\right]}{\sin \left(4 \omega_{0} \tau\right)+\sin \left[2\left(\omega-\omega_{0}\right) \tau\right]-\sin \left[2\left(\omega+\omega_{0}\right) \tau\right]},
$$




$$
\begin{aligned}
\left|a_{1}\right|^{2} & =\frac{-\sin \left[2\left(\omega+\omega_{0}\right) \tau\right]}{\sin \left(4 \omega_{0} \tau\right)+\sin \left[2\left(\omega-\omega_{0}\right) \tau\right]-\sin \left[2\left(\omega+\omega_{0}\right) \tau\right]}, \\
\left|a_{2}\right|^{2}+\left|a_{3}\right|^{2} & =\frac{\sin \left[4 \omega_{0} \tau\right]}{\sin \left(4 \omega_{0} \tau\right)+\sin \left[2\left(\omega-\omega_{0}\right) \tau\right]-\sin \left[2\left(\omega+\omega_{0}\right) \tau\right]} .
\end{aligned}
$$

It is now convenient to introduce the parameters

$$
\beta=4 \omega_{0} \tau \quad \text { and } \quad \nu=\frac{1}{2}\left[\frac{\omega}{\omega_{0}}-1\right] .
$$

To emphasize the role of the perturbation we are only going to consider $\omega>\omega_{0}$, thus $\nu>0$. Using the above parametrization, the form of the initial states that evolve into an orthogonal state is

$$
\left|\psi\left(t_{0}\right)\right\rangle=\left|a_{0}\right|\left|u_{0}\right\rangle+e^{i \phi_{1}}\left|a_{1}\right|\left|u_{1}\right\rangle+e^{i \phi_{2}}\left|a_{2}\right|\left|u_{2}\right\rangle+e^{i \phi_{3}}\left|a_{3}\right|\left|u_{3}\right\rangle
$$

where

$$
\begin{aligned}
\left|a_{0}\right|^{2} & =\frac{\sin (\nu \beta)}{\sin (\beta)+\sin (\nu \beta)-\sin [(1+\nu) \beta]} \\
\left|a_{1}\right|^{2} & =\frac{-\sin [(1+\nu) \beta]}{\sin (\beta)+\sin (\nu \beta)-\sin [(1+\nu) \beta]} \\
\left|a_{2}\right|^{2} & =\frac{\delta \sin (\beta)}{\sin (\beta)+\sin (\nu \beta)-\sin [(1+\nu) \beta]} \\
\left|a_{3}\right|^{2} & =\frac{(1-\delta) \sin (\beta)}{\sin (\beta)+\sin (\nu \beta)-\sin [(1+\nu) \beta]}
\end{aligned}
$$

with $\delta \in[0,1]$ and $\phi_{1}, \phi_{2}, \phi_{3} \in[0,2 \pi)$. For the right hand sides in (55) to be all non-negative quantities it is necessary that $\beta \geq \frac{\pi}{\nu+1}$ (note that, since $\beta$ is proportional to the orthogonality time, it is always a positive quantity). States with very large orthogonality time, and correspondingly very large $\beta$, are not interesting for our present purposes because those states are not useful in order to implement fast information-related tasks. Consequently, it is sensible to study the connection between quantum speed and entanglement by restricting the analysis only to states that do not have very large values of $\beta$. So we focus on the range of values $\beta \in\left[\frac{\pi}{\nu+1}, \pi\right)$. In order to calculate $\tau_{\min }$ in (3) we need the mean energy and standard deviation, which are given by

$$
E=4 \hbar \omega_{0}\left\{\frac{-\sin [(1+\nu) \beta]+(1+\nu) \sin (\beta)}{\sin (\beta)+\sin (\nu \beta)-\sin [(1+\nu) \beta]}\right\}
$$




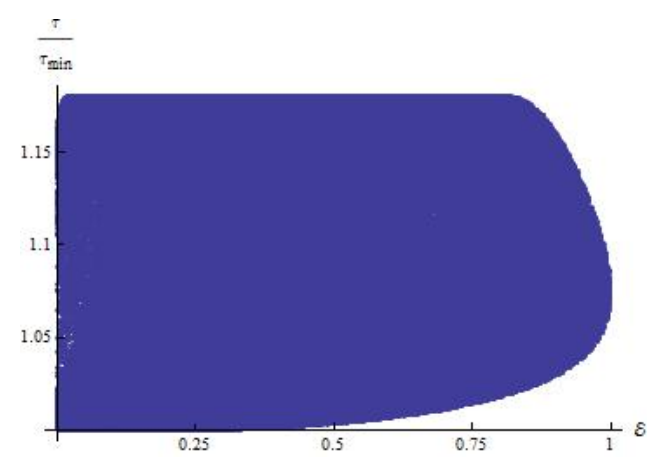

FIG. 7: Initial states that evolve into orthogonal states for $\nu=1.2$. All depicted quantities are dimensionless.

$$
\begin{aligned}
\triangle E= & 4 \hbar \omega_{0}\left\{\frac{-\sin [(1+\nu) \beta]+(\nu+1)^{2} \sin (\beta)}{\sin (\beta)+\sin (\nu \beta)-\sin [(1+\nu) \beta]}\right. \\
& \left.-\left(\frac{-\sin [(1+\nu) \beta]+(\nu+1) \sin (\beta)}{\sin (\beta)+\sin (\nu \beta)-\sin [(1+\nu) \beta]}\right)^{2}\right\}^{\frac{1}{2}} .
\end{aligned}
$$

Let us first discuss the special case $\beta=\pi$. In that instance, for general non-integer values of $\nu$, the only states that evolve to orthogonal states are maximally entangled and have $\left|a_{0}\right|^{2}=\left|a_{1}\right|^{2}=1 / 2$ and $\left|a_{2}\right|^{2}=\left|a_{3}\right|^{2}=0$. For integer values of $\nu$ the solution (55) is not valid. However, the correct solution of (51) in this case admits a parametrization similar to the $s$-case discussed previously. Indeed, the solutions for odd integer values of $\nu$ correspond precisely to the alluded $s$-case, and the correct parametrization for the states evolving into orthogonal ones is then given by (42) and (43). For even integer values of $\nu$ similar coefficients are obtained, but with $a_{0}$ and $a_{1}$ interchanged.

As already mentioned, we are going to focus on states with $\beta \in\left[\frac{\pi}{\nu+1}, \pi\right)$. The initial states that reach an orthogonal state are parameterized by the five parameters $\beta, \delta, \phi_{1}, \phi_{2}, \phi_{3}$. For general $\nu$-values the expressions for the entanglement and the quotient $\frac{\tau}{\tau_{\min }}$ become too cumbersome, and it is thus not possible to analytically obtain the boundaries of the physically allowed region in the $\left(\mathcal{E}, \frac{\tau}{\tau_{\min }}\right)$-plane corresponding to general states evolving to an orthogonal one. However, the shape of this region can be investigated numerically by recourse to a Monte Carlo-like procedure. In order to do that we numerically generate random initial states, corresponding to random values of the aforementioned parameters. We implemented this numerical procedure for several $\nu$-values. As an illustration, the region in the $\left(\mathcal{E}, \frac{\tau}{\tau_{\min }}\right)$-plane corresponding to $\nu=1.2$ is depicted in Figure 7 . 


\section{DISCUSSION AND COMPARISON BETWEEN THE INTERACTING AND NON-INTERACTING SCENARIOS}

We have explored the connection between the speed of quantum evolution, as given by the ratio $\tau / \tau_{\min }$, and quantum entanglement for quantum evolutions of two interacting qubits governed by the Hamiltonian (4). We have considered the particular instances that allow for an analytical treatment, and also provided some numerical results concerning more general cases. The expressions for $\tau_{\min }$ corresponding to the different cases investigated by us are summarized in the following table.

\begin{tabular}{|c|c|}
\hline Hamiltonian's Parameters & $\tau_{\text {min }}$ \\
\hline \hline$\omega=\omega_{0}$ & $\frac{\pi}{4 \omega_{0}}$ \\
\hline$\omega_{0}=0$ & $\frac{\pi}{2 \omega}$ \\
\hline$\omega=3 \omega_{0}$ & $\frac{\pi \sqrt{1-\cos (\beta)}}{8 \omega_{0}} \quad(\beta$-case $)$ \\
& $\frac{\pi(1-s)}{8 \omega_{0}}\left[\begin{array}{c}\left.\frac{-\sin [(1+\nu) \beta]+(\nu+1)^{2} \sin (\beta)}{\sin (\beta)+\sin (\nu \beta)-\sin [(1+\nu) \beta]}-\left(\frac{-\sin [(1+\nu) \beta]+(\nu+1) \sin (\beta)}{\sin (\beta)+\sin (\nu \beta)-\sin [(1+\nu) \beta]}\right)^{2}\right]^{-\frac{1}{2}}(E \geq \Delta E) \\
\end{array}\right.$ \\
\hline$\omega=(2 \nu+1) \omega_{0}$ & $\frac{\pi}{8 \omega_{0}}\left(\frac{\sin (\beta)+\sin (\nu \beta)-\sin [(1+\nu) \beta]}{-\sin [(1+\nu) \beta]+(1+\nu) \sin (\beta)}\right) \quad(\Delta E>E)$ \\
\hline
\end{tabular}

The cases $\omega=\omega_{0}$ and $\omega_{0}=0$ are special, because all initial states that do evolve into an orthogonal state reach this state in the minimum time $\tau=\tau_{\min }$. The physical reason behind this is that the Hamiltonians corresponding to these two cases have only two different eigenenergies (each one is two-fold degenerate). Consequently, as far as the quantum speed is concerned, they behave as two-level systems, for which the states evolve into an orthogonal state in a time-optimal fashion [1].

The $\omega=0$ case of the Hamiltonian can also be studied analytically and corresponds to a system constituted by non-interacting parts [18]. It is useful to provide a brief summary of its main features, in order to compare them with the ones corresponding to the interacting case investigated in the present contribution. As occurs in the $\omega=3 \omega_{0}$ case already analyzed in this paper, for the non-interacting scenario it is also convenient to consider two different cases of the characteristic equation (15) that determines the orthogonality time $\tau$. This 
equation can have two complex conjugate roots ( $\beta$-case) or it may have two real roots, one of them equal to -1 ( $s$-case). In general, it is seen that states of higher entanglement tend to be faster than states of lower entanglement, in the sense of having smaller values of $\tau / \tau_{\min }$.

The case $\omega=3 \omega_{0}$ is the one exhibiting the richest structure. The main results concerning this case are summarized in Figures 1-6. There is a general tendency that can be appreciated in these figures: states of low initial entanglement are those that tend to evolve in the fastest way. This means that the entanglement generated by the time evolution itself is able to enhance the quantum speed of initial states of relatively low entanglement.

As in the non-interacting case, it is useful to consider two different families of initial states: those for which the characteristic equation has two complex conjugate roots $(\beta$-case) and those for which this equation has two real roots, one of them equal to -1 ( $s$-case). As can be appreciated in Fig.1, $\beta$-case initial states of low entanglement tend to evolve faster than initial states of high entanglement. Among $\beta$-case states, those reaching the speed limit have relatively low entanglement values belonging in the interval $[0,1 / 4]$. These features of the $\beta$ case initial states are much more noticeable if, instead of considering the plane $\left(\mathcal{E}, \frac{\tau}{\tau_{\min }}\right)$, we consider the plane $\left(\langle\mathcal{E}\rangle, \frac{\tau}{\tau_{\min }}\right)$, where we do not represent the initial entanglement $\mathcal{E}$, but the time averaged entanglement $\langle\mathcal{E}\rangle$. This plane is represented in Fig.2. As already mentioned, the connection between entanglement and quantum speed is now much more evident. The physically allowed region, corresponding to initial states that reach an orthogonal state, is much smaller in Fig.2 than in Fig.1, and a clear trend associating low entanglement with high speed (in the sense of having an orthogonality time $\tau$ close to the minimum one $\tau_{\min }$ ) can be observed.

Symmetric states are particularly relevant with regards to the connection between entanglement and the quantum speed of evolution. This connection tends to be stronger for symmetric states than in the case of asymmetric ones. In fact, highly asymmetric states, where only one of the qubits actually evolves, can reach the speed limit even if no entanglement at all is involved (that is, even in the case of an initially separable state of a system of independent non-interacting qubits). The $\left(\mathcal{E}, \frac{\tau}{\tau_{\min }}\right)$-plane corresponding to initially symmetric $\beta$-case states is depicted in Fig.3. An interesting aspect of the data depicted in Fig. 3 is that the only $\beta$-case states saturating the speed bound have the same amount of entanglement, $\mathcal{E}=\frac{1}{4}$. These states are especially interesting because they define a family of energetically symmetric states with low entanglement (ESSLE) that saturate the speed 
bound.

The $\left(\mathcal{E}, \frac{\tau}{\tau_{\min }}\right)$ and $\left(\langle\mathcal{E}\rangle, \frac{\tau}{\tau_{\min }}\right)$ planes corresponding to initial $s$-case states are plotted, respectively, in Figures 5 and 6 . In contrast with what happens in the $\beta$-case, there are initially maximally entangled $s$-case states that achieve the speed limit. This seems to contradict the general trend associating initial states of low entanglement with high speed (in the sense of having $\tau / \tau_{\min }$ close to one). However, the $s$-states (even those with $\frac{\tau}{\tau_{\min }}=1$ ) have the largest possible orthogonality time $\tau$. That is, in terms of the absolute value of $\tau$ (as opposed to its value relative to $\tau_{\text {min }}$ ) the $s$-case states evolve in the slowest possible way.

Finally, let us consider more general values of $\omega$ and $\omega_{0}$. The (numerically determined) region in the $\left(\mathcal{E}, \frac{\tau}{\tau_{\min }}\right)$-plane corresponding to $\nu=1.2$ is shown in Figure 7 . We observe that the shape of this region differs appreciably from the corresponding region of the $\beta$-case for $\omega=3 \omega_{0}(\nu=1)$. However, the essential features exhibited by the fast states with low values of the quotient $\frac{\tau}{\tau_{\min }}$ remain the same. In particular, the maximum possible value of entanglement for which the optimal speed (in the sense of having $\tau / \tau_{\min }=1$ ) is reached is $1 / 4$. That is, all states that saturate the quantum speed limit have relatively low entanglement. We have performed a numerical study of other cases corresponding to other $\nu$-values and our results suggest that this occurs for all $\nu \geq 1$. Furthermore, we observe that the initial states of highest entanglement reaching the speed bound are always symmetric states. Moreover, for all symmetric states the maximum speed is reached only for one value of the entanglement, namely $1 / 4$.

\section{CONCLUSIONS}

We have studied the connection between entanglement and the speed of quantum evolution (as determined by the orthogonality time) for a system of two interacting qubits. We have performed a systematic analysis of this connection for all the initial states of this system that actually evolve into a state orthogonal to, and consequently fully distinguishable from, the initial one. The general trend observed is that, with the exception of some marginal special cases, only initial states with relatively low entanglement do achieve the quantum speed limit given by the minimum orthogonality time $\tau_{\min }$. In this sense, the present system exhibits a behavior complementary to the one observed in systems of non-interacting 
qubits, which are characterized by the opposite trend, where initial states with relatively high entanglement are those who tend to reach the minimum orthogonality time.

\section{Acknowledgments}

This work was partially supported by the MEC grant FIS2011-23526 (Spain), by the Projects FQM-2445 and FQM-207 of the Junta de Andalucia, the grant FIS2011-24540 of the Ministerio de Innovacion y Ciencia (Spain), by the Conselleria d'Educacio, Cultura i Universitats (CAIB), and by FEDER (EU).

[1] Margolus N and Levitin L B 1998 Physica D 120188

[2] Levitin L B and Toffoli T 2009 Phys. Rev. Lett. 103160502

[3] Giovannetti V, Lloyd S and Maccone L 2003 Europhys. Lett. 62615

[4] Giovannetti V, Lloyd S and Maccone L 2003 Phys. Rev. A 67052109

[5] Pfeifer P and Frölich J 1995 Rev. Mod. Phys. 67759

[6] Dodonov V V and Man'ko V I 1989 Invariants and the Evolution of Nonstationary Quantum Systems - Proc. P.N. Lebedev Physical Institute 183, Eds. Dodonov V V and Man'ko V I, New York: Nova, pp 3-101

[7] Fröwis F 2012 Phys. Rev. A 85052127

[8] Caneva T, Murphy M, Calarco T, Fazio R, Montangero S, Giovannetti V, and Santoro G E 2009 Phys. Rev. Lett. 103240501

[9] Kosinski P and Zych M (2006) Phys. Rev. A 73024303

[10] Luo S L and Zhang Z M 2005 Lett. Math. Phys. 711

[11] Luo S L 2004 Physica D 1891

[12] Sawyer R F 2004 Phys. Rev. A 70022308

[13] Andrecut M and Ali M K 2004 Journ. Phys. A: Math. Gen. 37 L157

[14] Andrecut M and Ali M K 2004 Int. Journ. Theor. Phys. 43969

[15] Brody D C 2003 Journ. Phys. A: Math. Gen. 365587

[16] Yurtsever U 2010 Physica Scripta 82035008 
[17] Oliveira V C G, Santos H A B, Torres L A M and Souza A M C 2008 Int. Journ. Quant. Inf. 6379.

[18] Zander C, Plastino A R, Plastino A and Casas M 2007 J. Phys. A: Math. Theor. 402861.

[19] Curilef S, Zander C and Plastino A R 2008 J. Phys.: Conf. Ser. 134 012003; Curilef S, Zander C and Plastino A R 2006 Eur. J. Phys. 271193

[20] Batle J, Casas M, Plastino A and Plastino A R 2005 Phys. Rev. A 72 032337; Batle J, Casas M, Plastino A and Plastino A R 2006 Phys. Rev. A 73 049904; Batle J, Borras A, Casas M, Plastino A and Plastino A R 2010 Phys. Rev. A 82 056302; Chau H F 2010 Phys. Rev. A 82 056301

[21] Borras A, Casas M, Plastino A R and Plastino A 2006 Phys. Rev. A 74022326

[22] Carlini A, Hosoya A, Koike T and Okudaira Y 2006 Phys. Rev. Lett. 96060503

[23] Brody D C and Hook D W 2006 J. Phys. A 39 L167; Brody D C and Hook D W 2007 J. Phys. A 4010949

[24] Bender C M, Brody D C, Jones H and Meister B K 2007 Phys. Rev. Lett. 98040403

[25] Borras A, Majtey A P and Casas M 2008 Phys. Rev. A 78022328

[26] Mostafazadeh A 2009 Phys. Rev. A 79014101

[27] Pati A K, Pradhan S and Agrawal P 2012 Quant. Inf. Proc. 11841

[28] Zhao B K, Deng F G, Zhang F S and Zhou H Y 2009 Phys. Rev. A 80052106.

[29] Borras A, Zander C, Plastino A R, Casas M and Plastino A 2008 Europhys. Lett. 8130007

[30] Borras A, Plastino A R, Casas M and Plastino A 2008 Phys. Rev. A 78052104

[31] Lloyd S 2003 Phys. Rev. Lett. 90167902

[32] Dodonov V V 2002 J. Opt. B: Quantum Semiclass Opt. 4 S98

[33] Ekert A and Knight P L 1995 Am. J. Phys. 63415

[34] Law C K and Eberly J H 2004 Phys. Rev. Lett. 92127903

[35] Karelin M U 2007 Opt. Spectros. 103193

[36] Naudts J and Verhulst T 2007 Phys. Rev. A 75062104 